\title{
RAPID INSPECTION OF PAVEMENT MARKINGS USING MOBILE LIDAR POINT CLOUDS
}

\author{
Haocheng Zhang ${ }^{\mathrm{a}}$, Jonathan $\mathrm{Li}^{\mathrm{a}}{ }^{\mathrm{b} *}$, Ming Cheng ${ }^{\mathrm{b}}$, Cheng Wang ${ }^{\mathrm{b}}$ \\ a Mobile Mapping Lab, Department of Geography and Environmental Management, University of Waterloo, 200 University Avenue \\ West, Waterloo, ON N2L 3G1, Canada - (h232zhan, junli)@uwaterloo.ca \\ ${ }^{\mathrm{b}}$ Fujian Key Laboratory of Sensing and Computing for Smart Cities, School of Information Science and Engineering, \\ Xiamen University, 422 Siming Road South, Xiamen, FJ 361005, China - (junli, chm99, cwang)@xmu.edu.cn
}

Commission I, ICWG I/Va

KEY WORDS: Mobile LiDAR, Point Cloud, Pavement Marking, Urban Road, Automated Detection

\begin{abstract}
:
This study aims at building a robust semi-automated pavement marking extraction workflow based on the use of mobile LiDAR point clouds. The proposed workflow consists of three components: preprocessing, extraction, and classification. In preprocessing, the mobile LiDAR point clouds are converted into the radiometrically corrected intensity imagery of the road surface. Then the pavement markings are automatically extracted with the intensity using a set of algorithms, including Otsu's thresholding, neighborcounting filtering, and region growing. Finally, the extracted pavement markings are classified with the geometric parameters using a manually defined decision tree. Case studies are conducted using the mobile LiDAR dataset acquired in Xiamen (Fujian, China) with different road environments by the RIEGL VMX-450 system. The results demonstrated that the proposed workflow and our software tool can achieve $93 \%$ in completeness, $95 \%$ in correctness, and $94 \%$ in F-score when using Xiamen dataset.
\end{abstract}

\section{INTRODUCTION}

Intelligent transportation systems (ITS) have been introduced for many years. The current development of ITS focuses on the driverless car, which is the most viable form of ITS. As a robotic vehicle that is capable of traveling between destinations without a human operator, the driverless car is driven not by human but the data. There are two principal data sources for the navigation of the driverless cars. The first one is the sensory input in terms of the surroundings of the driverless car. It can be acquired and collected by the radar, LiDAR sensor and video cameras. The second one is a prior highly detailed 3D map. Different from the traditional road map, this detailed 3D map is developed for the machine. The highly precise map is indispensable not only to allow a car to locate itself on the traffic lane but also enable a vehicle to take corresponding actions correctly (Guizzo, 2011).

To capture the world in 3D for autonomous vehicles, mobile laser scanning (MLS) technology is applied before the driverless cars hit the road. It is an effective and efficient method for acquiring highly accurate, precise, and dense georeferencing 3D topographic data (Puente et al., 2013). MLS systems are the mobile mapping systems based on the LiDAR, which capture 3D point clouds from the surrounding environment using profiling scanners. The detailed 3D point cloud data normally involves the important information of traffic lane such as lanes' geometry and boundary, lanes' types, speed limit and lane traversal information. The indispensable data helps the driverless car make solid decisions (Kent, 2015).

It is noted that majority of the information of lanes is represented by the road markings, thus, a variety of study has been carried out on the detection of road markings from point clouds. In general, the road markings have much higher reflectance than the unpainted road surface. Therefore, their relatively high intensity can be used for detecting the road markings. Smadja et al. (2010) implemented a simple threshold on intensity for detecting the road markings. Toth et al. (2008) selected an intensity value based on the intensity distribution in a search window as a global threshold for the extraction. Yang et al. (2012) extracted continuous edge lines and broken lane line markings successfully. Studies were also undertaken aiming at solving the problem caused by inconstant intensity. Chen et al. (2009) selected the intensity peaks along the scan line as lane marking points by using adaptive thresholding. Vosselman (2009) proposed a distance-dependent thresholding method to detect the road markings, and a connected components analysis to extract road markings. In other studies, the distancedependence of intensity was used in different ways. Guan et al. (2014) implemented multi-thresholding segmentation to extract road markings. The distribution of point density along the cross section was fitted to a Gaussian normal distribution function. The road surface points were segmented into some bins according to the estimated mean and standard deviation. Yu et al. (2015) applied a distance-dependent multi-thresholding segmentation in which the road surface points were segmented into the blocks along the road.

This study focuses on the extraction and recognition of road markings from MLS data for building the prior map of driverless car. The detailed research objectives include: (1) Completely extract the road markings from MLS data automatically with high accuracy in different environments, and (2) classify the road markings into thorough and detailed categories.

\footnotetext{
* Corresponding author
} 


\section{STUDY AREA AND DATASET}

The study area is located in the City of Xiamen. Xiamen is a port city on the southeast coast of mainland China. A complete survey of mobile laser scanning was carried out back and forth on Xiamen Island Ring Road on 23 April 2012 by a RIEGL VMX-450 system. The total length for one direction survey was around $10 \mathrm{~km}$. This primary road in Xiamen is characterized by numerous vehicles, trees, shafts (e.g., light poles and traffic poles). The majority of the road surface and road markings are in good condition. Seven samples of the survey data (i.e. the straight, curve roads and different types of road markings) were selected as the test dataset for evaluating the proposed method.

The average point density of the dataset is 7,000 points $/ \mathrm{m}^{2}$. The dataset was then converted into the format of LAS that is a standard in the laser scanning industry.

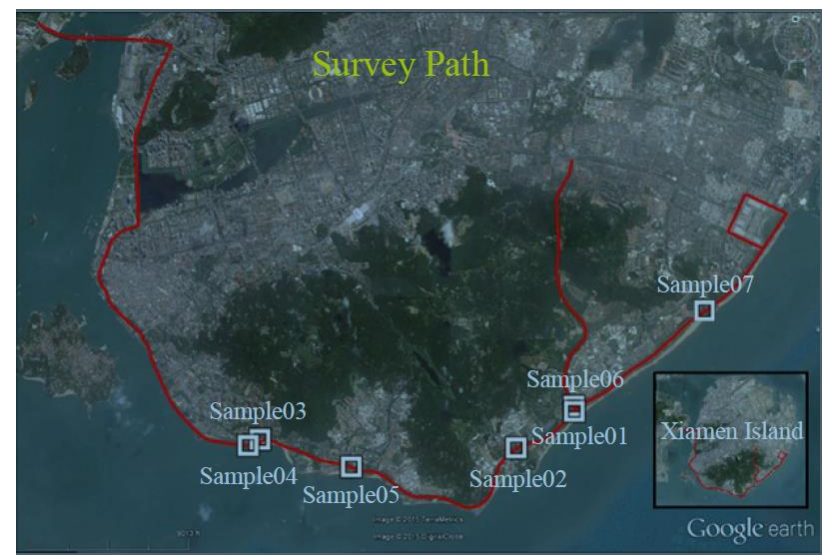

Figure 1. Study area (Island Ring Road, Xiamen, Fujian, China)

\section{METHODS}

The proposed method within this study consists of three phases, including preprocessing, road marking extraction, and road marking classification.

\subsection{Preprocessing}

The raw point clouds are preprocessed in two steps to reduce the volume of the data and overcome the problem resulting from the uneven distribution of intensity data.

The first step is road surface detection. The non-ground removal is implemented to extract ground points from the MLS data. The ground surface points are rasterized into a digital terrain model (DTM) by inverse distance weighted (IDW) interpolation.

A voxel-based upward growing method (Yu, 2015) is employed to segment the raw MLS data into ground points and nonground points. This method partitions point cloud data into an octree structure with a voxel size. For each voxel, it expands to its 9-neighbour upward voxels, and then the growing scheme expends until it reaches the top boundary. If the elevation of the top voxel is smaller than the predefined threshold, the cluster of these voxels is referred to the ground. The point clouds in these voxels are labelled as ground points. Otherwise, the point clouds will be categorized as non-ground points. In the removal process, all the non-ground points are removed from raw point clouds. The volume of the ground points concentrates $60 \%$ of the raw MLS data. To further reduce the data volume and improve the computational efficiency, the ground points will be rasterized into the DTM by IDW interpolation. With the IDW interpolation, the grey value of a grid is interpolated with its neighbours:

$$
z(x)=\sum_{k=1}^{n} w_{k} z_{k} / \sum_{\mathrm{k}=1}^{n} w_{k}, w_{k}=1 / d_{k}^{2}
$$

where $w_{k}$ is the weight of the $k$-th point within the grid, as the function of distance $d_{k} ; z_{k}$ is the $k$-th point grey value; $n$ is the number of points in a grid.

The main features of the road surface are characterised by its smoothness and connectedness. In general, the surface of the grass is rougher than the road surface. Additionally, it is clear that the existence of a curb would result in a sudden change in the height on the road boundary. Therefore, a high-pass filter is applied to the DTM, and the absolute values of the road surface will be much lower than the values of the other surfaces.

Intensity of Pure Road Marking vs. Cosine of the Scan Angle Rank

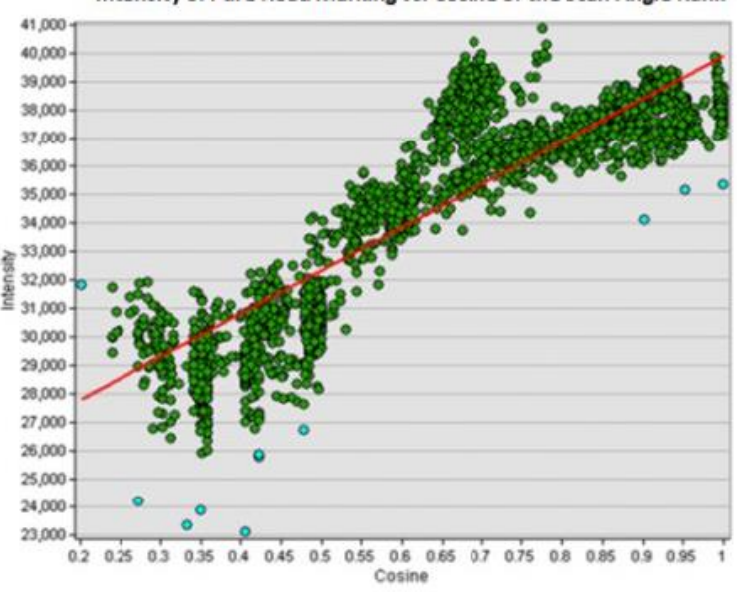

Figure 2. Cosine of the scan angle rank vs. intensity

The second step is the intensity correction and enhancement. The intensity value of the ground surface points are corrected by the scan angle rank, following by the IDW interpolation. The intensity values of the point clouds have a positive correlation with cosines of incidence angles, as shown in Figure 2. Thus, the linear regression model generated from the intensity image can be used to correct the intensity value of MLS data. The formula of the linear regression model is presented as below:

$$
y=15115 \cdot x+24794
$$

\subsection{Road Marking Extraction}

The Otsu's thresholding method is applied to extract road markings. It segments the image automatically with the optimum threshold that helps minimize the within-class variance. It is assumed that the image is bimodal and the illumination is uniform, therefore the bimodal brightness can be determined based on the differences of the materials' properties.

The road surface area is preserved in the intensity images in the format of either asphalt pavements or the road markings. With the assistance of the intensity correction, the illumination in the image is uniform. Thus, the corrected intensity image is able to meet the requirements of the Otsu's thresholding method, and the global thresholding process can be employed. 


\subsection{Road Marking Classification}

3.3.1 Road Marking Segmentation: The first step of road marking classification is dividing the extracted road marking into segments, where a 4-neighbor region growing segmentation is employed. Figure 3 shows an extracted road marking and the segmentation result, in which the noise and little clusters are removed in the procedure of region growing.

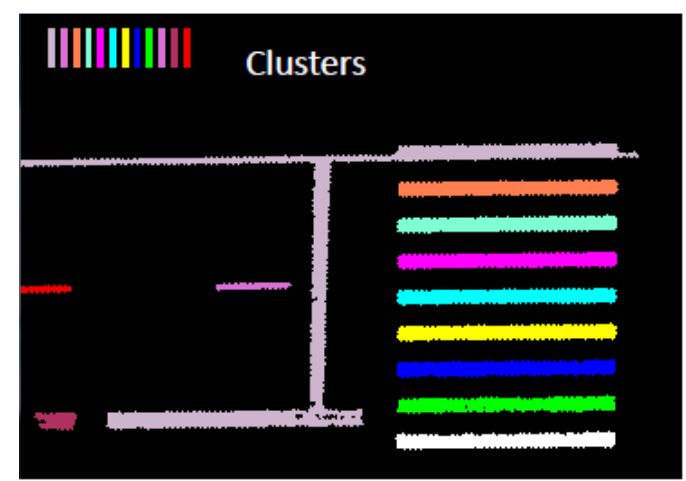

Figure 3. Region growing segmentation

The large road marking in Figure 3 should be segmented into a few road markings to guarantee the subsequent recognition. Taking the width and junction into consideration, the large road marking can be classified into two types: the thin road marking and the wide road marking. The segmentation method consists of two steps: distinguishing thin and wide road markings, and splitting road markings at junctions. The neighbour-counting filtering is applied to detect the thin, wide road markings and their junctions.

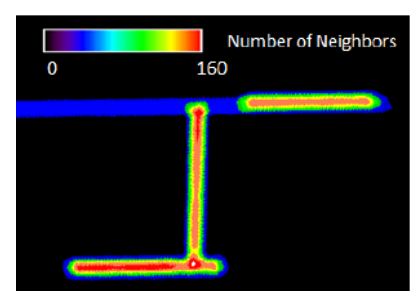

(a)

(c)

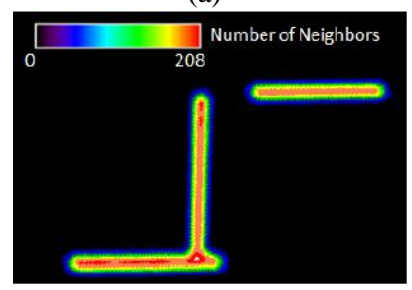

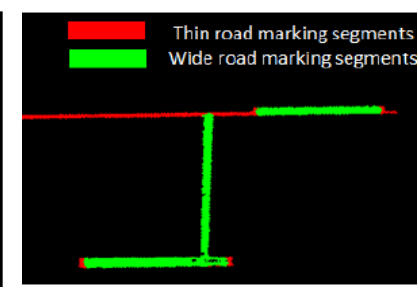

(b)

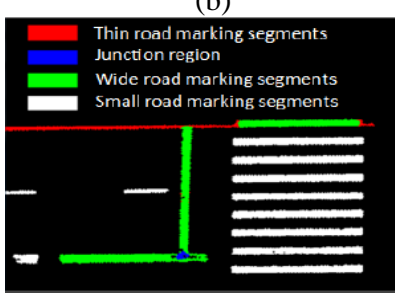

(d)
Figure 4. Large road marking segmentation: (a) Neighbourcounting image, (b) wide and thin road marking, (c) neighbourcounting image of wide road marking, and (d) segmentation result of large road marking.

3.3.2 Feature Extraction: After the road marking is partitioned into segments, geometric parameters of marking segments will be calculated. Four parameters were employed including area, perimeter, estimated width, and orientation. The estimated width is the function of area and perimeter:

$$
\text { width }=2 * \text { area } / \text { perimeter }
$$

Although this estimated value is not the true width of the segment, it can indicate the thinness of road marking. Based on area and width, rectangular markings can be detected and recognized, but irregular markings having similar area and width may be misclassified. In this case, a minimum bounding rectangular (MBR) is derived to present the extent of each road marking. According to the width of MBRs, road markings can be classified into thin MBRs (e.g., zebra strip and broken line) and wide MBRs (arrow, diamond, character, and number).

Based on the MBRs, the main angle of a road marking can be calculated.

3.3.3 Decision-Tree Based Classification: A decision tree is designed and developed for the classification. The hierarchical tree of road marking categories that developed in this study is illustrated in Figure 5. According to the People's Republic of China National Standards: Road Traffic Marking (2009), the road markings in Xiamen dataset can be classified into two categories: longitudinal and transverse marking. The orientation of road marking segments can be measured by the main angle of MBRs. Transverse and longitudinal markings have a high and low variance of the main angle in a section of the road respectively. The difference of orientation variance is adopted as the rule for the first level of the decision tree. At the second level, longitudinal marking segments are separated into two groups based on the MBR width. In the third level, if the width of the segment exceeds $45 \mathrm{~cm}$, it is refer to a wide MBR (i.e. road arrow or non-road arrow); otherwise, it is referred to a thin MBR (i.e. strip of zebra crossing, broken line or continuous line). In the fourth level, zebra crossings, broken lines, continuous lines, road arrows and non-road arrows are further classified into specific subclasses based on their area and estimated width.

\section{RESULTS AND DISCUSSION}

\subsection{Preprocessing}

Preprocessing aims to compress the large-sized data and balance the uneven distribution of intensity data prior to the extraction of the road markings. The ground removal result is shown in Figure 6.
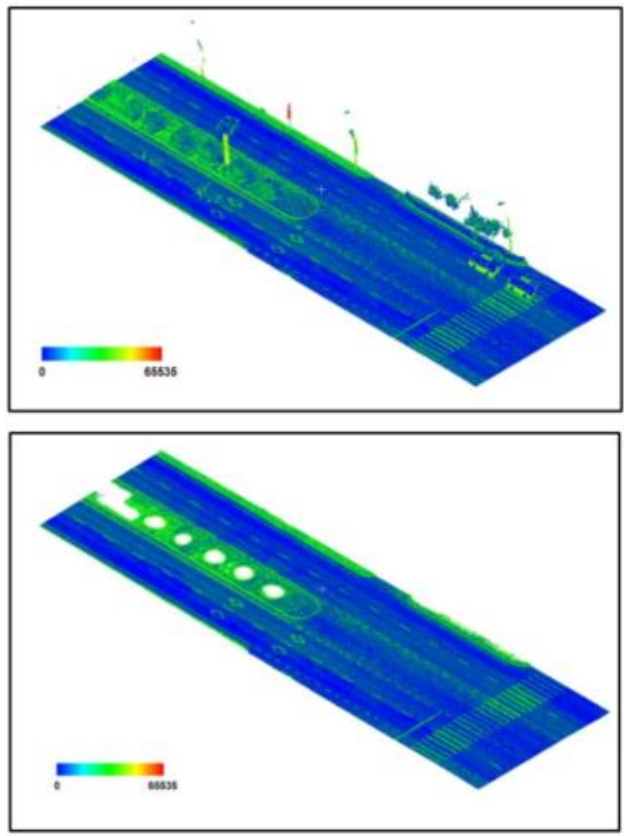

Figure 6. MLS data and ground surface points 


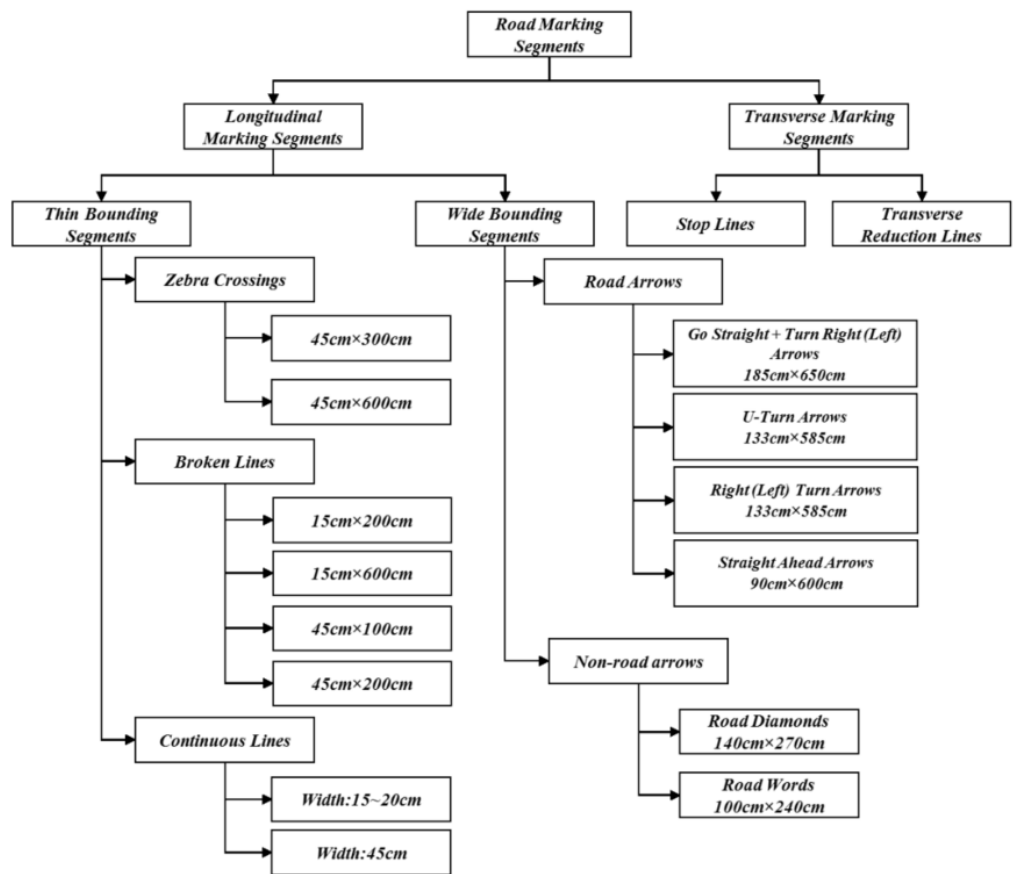

Figure 5. A hierarchical tree of the road marking categories

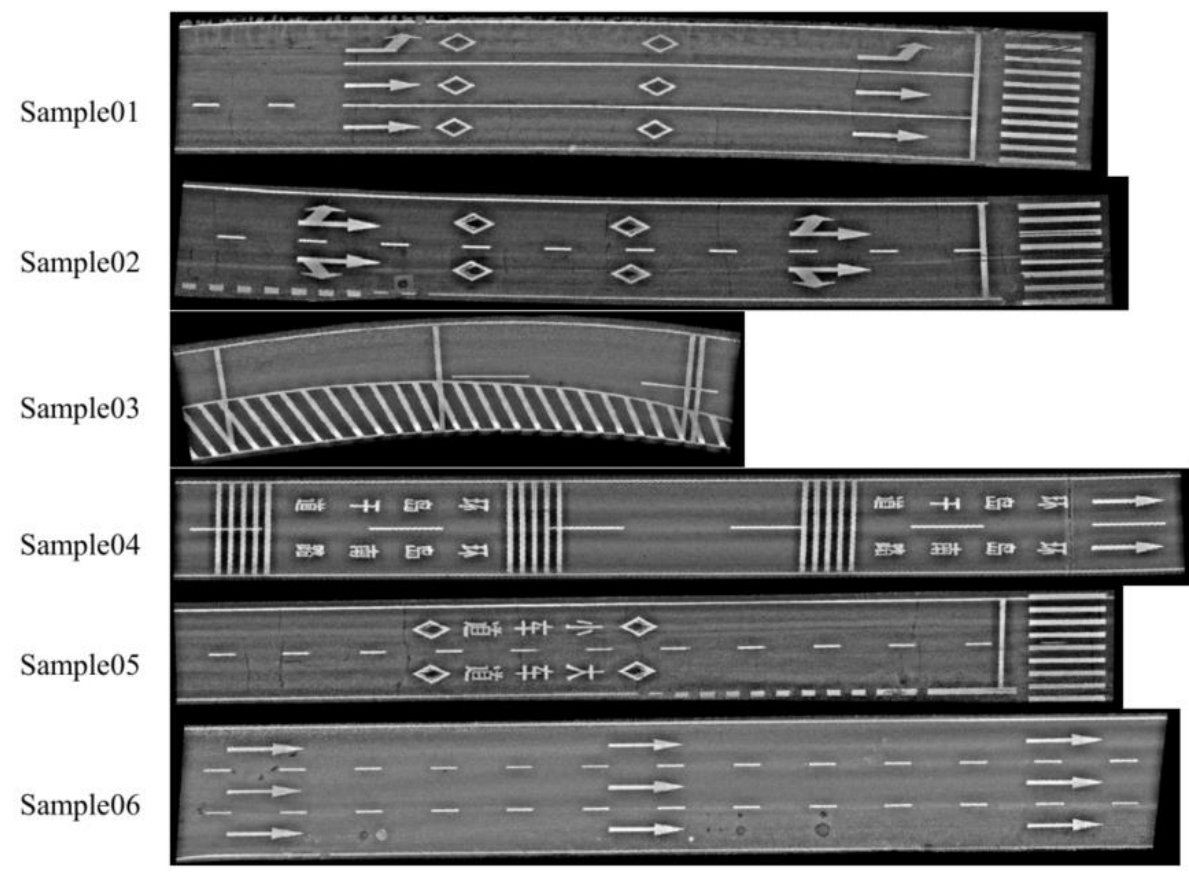

Figure 7. Intensity image after intensity correction

\begin{tabular}{|l|r|r|r|r|r|r|}
\hline Performance (\%) & Sample01 & Sample02 & Sample03 & Sample04 & Sample05 & Sample06 \\
\hline Completeness & 89.42 & 93.36 & 94.19 & 93.88 & 90.80 & 92.03 \\
\hline Correctness & 96.02 & 93.55 & 96.06 & 95.60 & 97.50 & 95.12 \\
\hline F-Score & 92.61 & 93.45 & 95.11 & 94.73 & 94.03 & 93.55 \\
\hline
\end{tabular}

Table 1. Quantitative assessment using completeness, correctness and F-Score

\begin{tabular}{|l|r|r|r|r|r|r|r|r|}
\hline Sample & \multicolumn{4}{|c|}{ Sample 01 } & \multicolumn{4}{|c|}{ Sample 05 } \\
\hline Method & Chen & Guan & Yu & Proposed & Chen & Guan & Yu & Proposed \\
\hline Completeness (\%) & 75 & 86 & 93 & 89 & 71 & 89 & 93 & 91 \\
\hline Correctness (\%) & 91 & 90 & 92 & 96 & 92 & 91 & 91 & 98 \\
\hline F-Score $\quad(\%)$ & 82 & 88 & 93 & 92 & 80 & 90 & 92 & 94 \\
\hline
\end{tabular}

Table 2. Quantitative evaluation results of different road marking extraction methods 


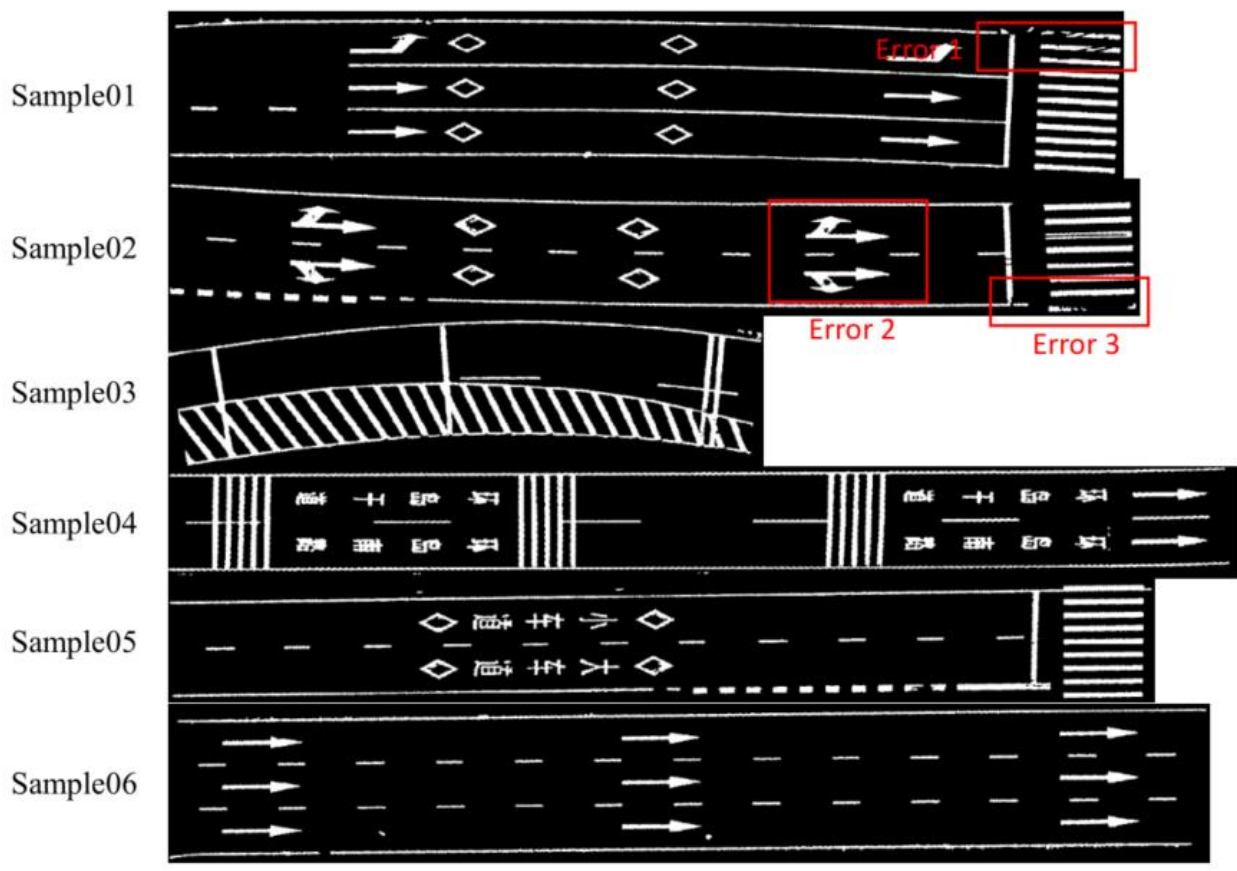

Figure 8. Results of road marking extraction based on Otsu thresholding

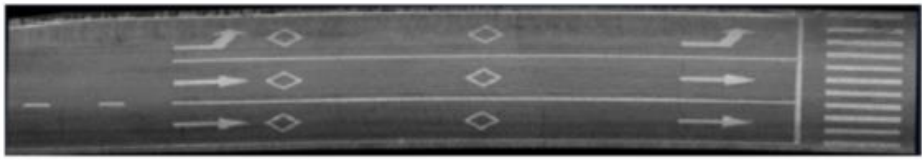

(a)

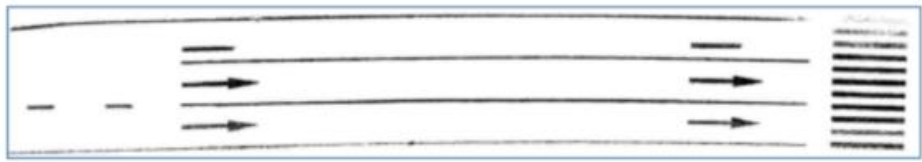

(b)

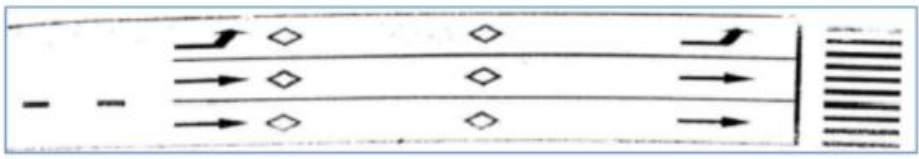

(c)

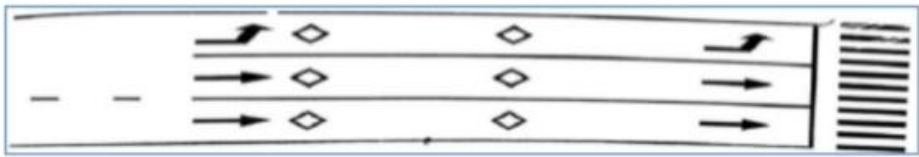

(d)

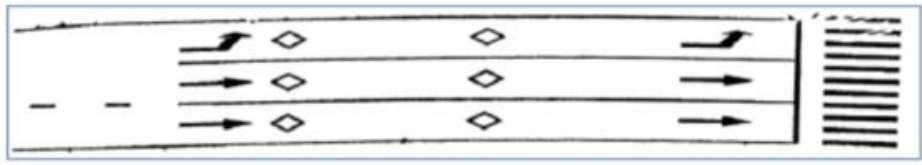

(e)

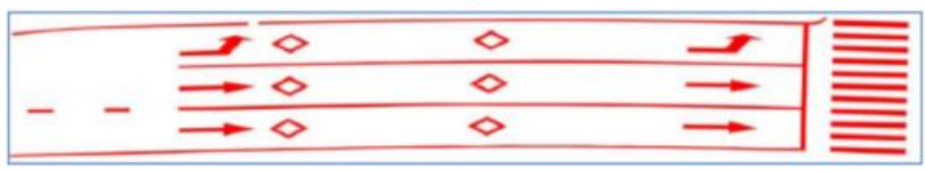

(f)

Figure 9. Extracted road markings from Sample 01 dataset: (a) road surface points, (b) Chen's method result, (c) Guan's method result, (d) Yu's method result, (e) proposed method result, and (f) manually labelled reference data (Adapted from: Yu, 2015). 


\begin{tabular}{|c|c|c|}
\hline Legends & & \\
\hline Transverse roadmarking: & Stopline & Transerve reduction line \\
\hline Continuous line: & Continuous line 1 & Continuous line 2 \\
\hline Thin Rectangle: & Broken Line 1 & Broken Line 2 \\
\hline & Broken Line 3 & Broken Line 4 \\
\hline & Zebra Crossing & \\
\hline Wide Rectangle: & Road Arrows 1 & Road Arrows 2 \\
\hline & Road Arrows 3 & \\
\hline & Road Words & Road Diamond \\
\hline Others: & Others & \\
\hline
\end{tabular}

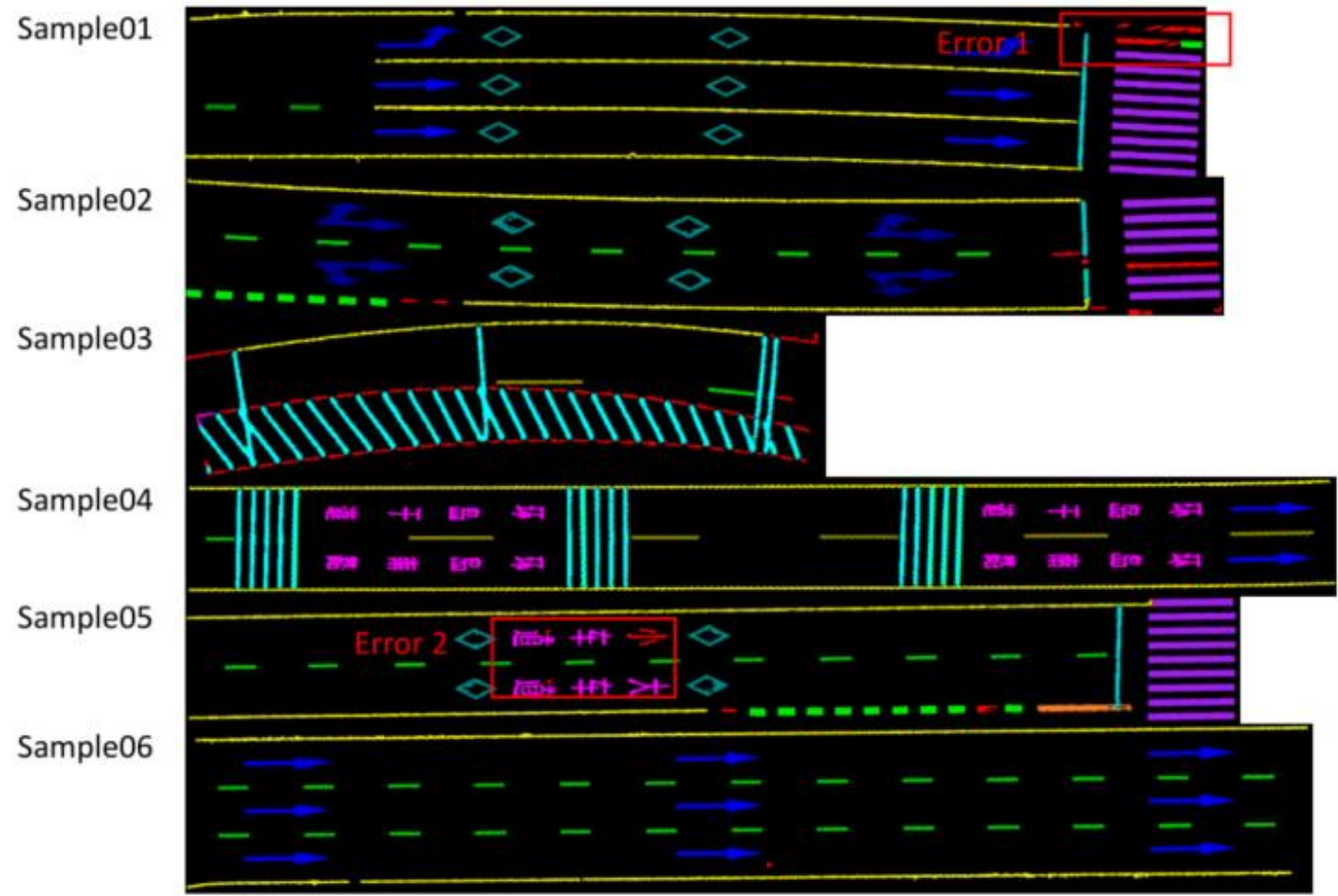

Figure 10. Results of road marking classification

In order to extract the road marking, a scan-angle-rank-based intensity correction was used to correct various intensity values caused by different incidence angles. The corrected intensity images are presented in Figure 7. It is evident that the contrast between road markings and the pavements was enhanced; therefore the road markings became more detectable.

\subsection{Road Marking Extraction}

Based on the corrected intensity image, the Otsu's thresholding was used to extract the road marking, and the results are shown in Figure 8. It is identified that majority of the road markings were extracted but with three errors. Errors 1 and 3 are caused by lacking of sufficient laser points, and error 2 is the false negative located in the inner of the road arrows.

As shown in the quantitative assessment (see Table 1), the proposed road marking extraction is capable to achieve $93 \%$ complete-ness, $95 \%$ correctness and $93 \%$ F-Score. The rate of completeness is smaller than the correctness's in each sample, indicating that some marking pixels were misclassified into the pavements. Due to the decay of the road markings, the sizes of manually labelled references are bigger than the damaged road markings'. Therefore, the performance of proposed method was underestimated in the result.

A comparative study was carried out between the proposed method and Chen's (Chen et al., 2009), Guan's (Guan et al., 2014) and Yu's methods (Yu et al., 2015), and the results are shown in Figure 9. The performance of these four methods is evaluated in a quantitative way, using three variables, i.e. completeness, correctness and F-score (see Table 2). It is identified that the proposed method outmatches Chen's and Guan's methods while it is inferior to Yu's method in terms of the completeness.

\subsection{Road Marking Classification}

The marking segments were classified into categories based on the decision tree. All kinds of the road markings in the samples 
are illustrated in Figure 10. It is identified that majority of the segments were classified into correct categories. Nevertheless, three problems arose in the classification.

Aiming at these unclassifiable segments, caused by marking decay, a rectification method can correct these misclassifications. The basic idea is that these unclassified segments should be reclassified according to the confessedly recognized road marking segments. Based on these correctly classified road-marking segments, the near unclassified segment can be set into the closest road marking. Although the damaged road marking cannot be classified correctly by its geometric features, it still has a strong spatial relationship with its congeneric markings.

In order to identify Chinese characters, successfully separating the strokes becomes the crucial point. One solution is to expand the segments of strokes to generate them to become one connected region, and then detected as one character correctly. The dilation, as one of the basic operators in the area of mathematical morphology, was tested to connect separate strokes. After region expanding, the strokes in one connect area can be classified as one character.

\section{CONCLUSION}

Majority of the existing MLS point clouds-based road marking extraction methods are based on the application of global intensity filtering and multi-thresholding segmentation. However, these methods could be greatly influenced by the unevenly distributed intensity. This study has highlighted that the application of the scan-angle-rank-based intensity correction and the large-size high-pass filtering have the potential to significantly reduce the in-class variance of road markings and pavements. In addition, based on the comprehensive prior knowledge, a shape-based hierarchical tree developed in this study is capable to undertake the classification in an efficient and systematic manner. Based on the case study results, the developed workflow is capable of rapid extraction and classification of the road markings in the MLS point clouds.

\section{ACKNOWLEDGEMENTS}

This work was supported in part by the National Natural Science Foundation of China under Grant 41471379 and in part by Fujian Collaborative Innovation Centre for Big Data Applications in Governments.

\section{REFERENCES}

Chen, X., Stroila, M., and Wang, R., 2009. Next generation map marking: geo-referenced ground-level LiDAR point clouds for automatic retro-reflective roadfeature extraction. In: 17th ACM SIGSPATIAL International Conference on Advances in Geographic Information System, Seattle, WA, USA, pp.4-6.

Guan, H., Li, J., Yu, Y., Wang, C., Chapman, M., Yang, B., 2014. Using mobile laser scanning data for automated extraction of road markings. ISPRS Journal of Photogrammetry and Remote Sensing, 87, pp.93-107.

Guizzo, E., 2011. How Google's self-driving car works. http:// spectrum.ieee.org/automaton/robotics/artificial-intelligencel how-google-self-driving-car-works/
L. Kent, 2015. Autonomous cars can only understand the real world through a map. http://360.here.com/2015/04/16/ autonomous-cars-can-understand-real-world-map/

Puente, I., González-Jorge, H., Martínez-Sánchez, J., and Arias, $\mathrm{P}, 2013$. Review of mobile mapping and surveying technologies. Measurement, 47, pp.2127-2145.

Smadja, L., Ninot, J., and Gavrilovic, T., 2010. Road extraction and environment interpretation from LiDAR sensors. ISPRS Archives, 38, pp.281-286.

Toth, C., Paska, E., and Brzezinska, D., 2008. Using road pavement markings as good control for LiDAR data. ISPRS Archives, 37 (B1), pp.189-196.

Vosselman, G., 2009. Advanced point cloud processing, In: Photogrammetric Week'09, Stuttgart, Germany, pp.137-146.

Yang, B., Fang, L., Li, Q., and Li, J., 2012. Automated extraction of road markings from mobile LiDAR point clouds, Photogrammetric Engineering \& Remote Sensing, 78(4), pp.331-338.

Yu, Y., Li, J., Guan, H., Jia, F. and Wang, C., 2015. Learning hierarchical features for automated extraction of road markings from 3-D mobile LiDAR point clouds. IEEE Journal of Selected Topics in Applied Earth Observations and Remote Sensing, 8(2), pp.709-726. 